\title{
Programme scientifique Wissenschaftliches Programm
}

\section{Programme scientifique Wissenschaftliches Programm}

\author{
83e Réunion annuelle de la Société Suisse de \\ Dermatologie et Vénéréologie \\ Genève, 27-29 septembre 2001 \\ Centre Médical Universitaire \\ 1 , rue Michel-Servet
}

83. Jahresversammlung der Schweizerischen Gesellschaft für Dermatologie und Venerologie Genf, 27.-29. September 2001

Centre Médical Universitaire

1 , rue Michel-Servet

Thème principal:

Les traitements topiques

Jeudi, 27 septembre 2001

Donnerstag, 27. September 2001

\subsection{0-16.00 h Ateliers}

Séminaire d'histopathologie cutanée: réactions cutanées médicamenteuses

P. Chavaz

Salle de microscopie, étage 1

Dermatoscopie: quiz

R. Braun

Auditoire B400, étage 1

Biotechnologie et plaies chroniques: état des lieux et

Médecine factuelle en dermatologie: analyse de la littérature des plaies chroniques

D. Salomon, L. Naldi, E. Laffitte

Auditoire A250, étage 1

Diagnostic et traitement des lymphomes cutanés: mise à jour

L. French

Auditoire D60, étage 1

Trichologie: mise à jour

R. Trüeb, M. Harms

Auditoire C150, étage

Vendredi, 28 septembre 2001

Freitag, 28. September 2001

8.30-9.00 h Inscriptions, montage des posters

9.00-9.30 h Le point sur les rétinoïdes topiques J.-H. Saurat, Genève

9.30-10.00 h Update on Topical Steroids C. Surber, Bâle

10.00-10.30 h Update on Topical Vitamin D P. van de Kerkhof, Nijmegen

10.30-11.00 h Update on Topical Immunostimulants U.R. Hengge, Essen

$11.00-11.30 \mathrm{~h}$ Pause, visite des posters et exposition industrielle

11.30-12.00 h Update on Topical Immunosuppressors T. Luger, Münster

12.00-12.30 h Présentations des cas de la Clinique de Bâle
12.30-14.00 h Déjeuner au restaurant «La Marmitière»

14.00-15.00 h Communications libres I (C1-C6)

C1 Les génodermatoses dans le miroir du génome humain D. Hohl, Lausanne

C2 Expression von $\mu$-Opiat-Rezeptoren und $\beta$-Endorphin in peripheren nichtmyelinisierten Nervenendigungen in der Haut P.L. Bigliardi, M. Bigliardi-Qi, L. Sumanovski, S. Büchner, T. Rufli, Basel/Schaffhausen

C3 Des liposomes comme modèles de membranes cellulaires pour tester l'effet filtre et la photoprotection dus à des molécules lipophiles

C. Tran, O. Sorg, L. Didierjean, J.-H. Saurat, Genève

$\overline{\mathrm{C} 4}$ Allergenität von prozessiertem Sellerie: DBPCFC mit Selleriegewürz und gekochtem Sellerie

B.K. Ballmer-Weber, S. Vieths, C. Pompei,

D. Lüttkopf, B. Wüthrich, Zürich

C5 Tissue engineering cutané tridimensionnel pour plaies chroniques et aiguës

A.-A. Ramelet, C. Scaletta, S. Rocque, P. Hohfeld,

N. Burri, L. Laurent-Applegate, Lausanne

C6 Autologous Suction Blister Transplantation for Chronic Leg Ulcers

U. Costanzo, L.R. Braathen, Berne

15.00-15.30 h Présentations des cas de la Clinique de Lausanne

15.30-16.00 h Pause, visite des posters et exposition industrielle

16.00-16.15 h Présentations des cas de la Division de dermatologie d'Aarau

16.15-18.30 h Assemblée générale de la SSDV

$20.00 \mathrm{~h}$ Cocktail et banquet au Bâtiment des Forces Motrices, 2, place des Volontaires (bus 1, 2,10,13,16: arrêt Stand)

Samedi, 29 septembre 2001

Samstag, 29. September 2001

8.30-9.00 h Présentations des cas de la Clinique de Zurich

9.00-10.00 h Révision des cas de dermatopathologie

Discussion et commentaires didactiques des biopsies de réactions cutanées médicamenteuses présentées le jeudi après-midi au microscope pour les médecins en formation et les praticiens par les dermatopathologues des cliniques universitaires et des laboratoires privés

10.00-10.30 h Pause, visite des posters et exposition industrielle

10.30-11.00 h Présentations des cas de la Policlinique de la Ville de Zurich

11.00-12.00 h Communications libres II (C7-C12)

C7 Charakteristische «Salz-und-Pfeffer»-artige Pigmentverteilung innerhalb der Lentigines bei einer Familie mit Carney-

Komplex: Ein neues klinisch-dermatoskopisches diagnostisches Merkmal

\section{J. Izakovic, P.H. Itin, Basel}

C8 Lokoregionäre Hautmanifestationen und primär inflammatorische Formen von Mammakarzinomen: Schlüsselveränderungen für Diagnose und Behandlung der kutanen Komplikationen P.H. Itin, M.D. Anliker, S. Borelli, A. Schönenberger, Aarau 


\section{Programme scientifique \\ Wissenschaftliches Programm}

C9 Das benigne Lymphangioendotheliom: Eine klinisch-pathologische Untersuchung an 4 Fällen

D.V. Kazakov, H. Kutzner, M. Graf, G. Burg,

W. Kempf, Zürich/Friedrichshafen

C10 Détermination par immunohistochimie de Melan-A/MART-1 comme facteur pronostique dans le mélanome

M. Berset, J.-P. Cerottini, D. Guggisberg, P. Romero,

F. Burri, D. Rimoldi, R.G. Panizzon,

Lausanne/Epalinges

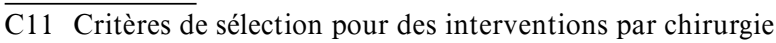
micrographique

C. Tschanz, A. Skaria, R. Braun, D. Salomon, Genève

C12 Dynamique tumorale et de lambeau A.M. Skaria, Vevey

12.00-12.30 h Présentations des cas de la Clinique de Berne

12.30-13.30 h Buffet, visite des posters et exposition industrielle

13.30-13.45 h Présentations des cas de l'Unité d'allergologie de Genève

13.45-15.05 h Communications libres III (C13-C21)

C13 Epidemiologie der Herpes-simplex-Infektion (HSV-1 und -2) in unterschiedlichen Schweizer Kollektiven

S. Lautenschlager, K. Quack, R. Weber,

A. Eichmann, Zürich

C14 Recensement des dermatophytes isolés à Lausanne de 1993 à 2000

M. Monod, S. Jaccoud, C. Zaugg, B. Léchenne,

F. Baudraz, R.G. Panizzon, Lausanne

C15 Pityriasis rosea: Eine Analyse des klinischen Spektrums anhand von 795 Fällen

J. Haintz, S. Büchner, Basel
C16 Infections à herpèsvirus simplex et traitement de la dermite atopique par le tacrolimus topique

J. Lübbe, C. Tschanz, J.-H. Saurat, Genève

C17 Pustulose érosive du scalp: traitement par tacrolimus topique E. Laffitte, G. Kaya, V. Piguet, J.-H. Saurat, Genève

$\overline{\text { C18 Imiquimo }}$ 5\% Crème (Aldara ${ }^{\circledR}$ ) ist unwirksam bei therapieresistenter Alopecia areata des Haarbodens U. Hesterberg, L. Böhlen, T. Hunziker, Bern

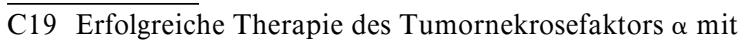
Etanercept bei pustulöser Exazerbation einer mutilierenden Psoriasis arthropathica nach Absetzen von Cyclosporin A J. Kamarachev, P. Lor, A. Forster, C. Burg, F.O. Nestle, Zürich

C20 Effet de hautes doses d'immunoglobulines intraveineuses dans le syndrome de Stevens-Johnson: étude rétrospective multicentrique

C. Prins, C. Vittorio, R.S. Padilla, R. Hunziker, P.H. Itin, J. Förster, E.-B. Bröcker, J.-H. Saurat, L. French, Genève/Philadelphie/Albuquerque/Berne/ Aarau/Berlin/Würzburg

C21 Traitement de la vulvodynie par antihistaminiques dans un contexte d'urticaire factice: étude prospective dans le cadre de la consultation de dermatogynécologie

G. Vecchietti, L. Germanier, J. Lübbe, A. Valiton, M. Harms, Genève

15.05-15.45 h Présentations des cas de la Clinique de Genève $15.45 \mathrm{~h}$ Clôture du Congrès 